\title{
Structural insights into alginate binding by bacterial cell-surface protein.
}

\section{$\operatorname{AUTHOR}(\mathrm{S})$ :}

Temtrirath, Kanate; Murata, Kousaku; Hashimoto, Wataru

\section{CITATION:}

Temtrirath, Kanate ...[et al]. Structural insights into alginate binding by bacterial cell-surface protein.. Carbohydrate research 2015, 404: 39-45

\section{ISSUE DATE:}

2015-03-02

URL:

http://hdl.handle.net/2433/196076

\section{RIGHT:}

(c) 2014 Elsevier Ltd. NOTICE: this is the author's version of a work that was accepted for publication in Carbohydrate Research. Changes resulting from the publishing process, such as peer review, editing, corrections, structural formatting, and other quality control mechanisms may not be reflected in this document. Changes may have been made to this work since it was submitted for publication. A definitive version was subsequently published in

Carbohydrate Research, 404 (2015) doi:10.1016/i.carres.2014.11.008; This is not the published version. Please cite only the published version.; この論文は出版社版でありません。引用の際には出版社版をご碓認ご利用ください。 
MS: for Carbohydr. Res.

Title: Structural insights into alginate binding by bacterial cell-surface protein

Authors: Kanate Temtrirath ${ }^{\mathrm{a}}$, Kousaku Murata ${ }^{\mathrm{a}, \mathrm{b}}$, Wataru Hashimoto ${ }^{\mathrm{a}, *}$

Affiliations: ${ }^{a}$ Laboratory of Basic and Applied Molecular Biotechnology, Division of Food Science and Biotechnology, Graduate School of Agriculture, Kyoto University, Gokasho, Uji, Kyoto 611-0011, Japan.

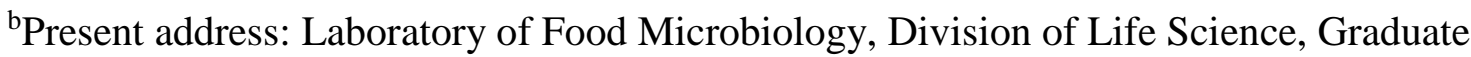
School of Science and Engineering, Setsunan University, Neyagawa, Osaka 572-8508, Japan.

*Corresponding author: W. Hashimoto

Laboratory of Basic and Applied Molecular Biotechnology, Division of Food Science and Biotechnology, Graduate School of Agriculture, Kyoto University, Gokasho, Uji, Kyoto 611-0011, Japan

Tel. +81-774-38-3756. Fax +81-774-38-3767

E-mail:whasimot@kais.kyoto-u.ac.jp 
Abstract: A gram-negative Sphingomonas sp. strain A1 inducibly forms a mouth-like pit on the cell surface in the presence of alginate and directly incorporates polymers into the cytoplasm via the pit and ABC transporter. Among the bacterial proteins involved in import of alginate, a cell-surface EfeO-like Algp7 shows an ability to bind alginate, suggesting its contribution to accumulate alginate in the pit. Here, we show identification of its positively charged cluster involved in alginate binding using X-ray crystallography, docking simulation, and site-directed mutagenesis. The tertiary structure of Algp7 was determined at a high resolution (1.99 ^) by molecular replacement, although no alginates were included in the structure. Thus, an in silico model of Algp7/oligoalginate was constructed by docking simulation using atomic coordinates of Algp7 and alginate oligosaccharides, where some charged residues were found to be potential candidates for alginate binding. Site-directed mutagenesis was conducted and five purified mutants K68A, K69A, E194A, N221A, and K68A/K69A were subjected to a binding assay. UV absorption difference spectroscopy along with differential scanning fluorimetry analysis indicated that K68A/K69A exhibited a significant reduction in binding affinity with alginate than wild-type Algp7. Based on these data, Lys68/Lys69 residues of Algp7 probably play an important role in binding alginate.

Keywords: Sphingomonas; alginate-binding protein; EfeO; X-ray crystallography; docking simulation

\section{Introduction}

Alginate is a linear acidic polysaccharide composed of $\beta$-D-mannuronate $(\mathrm{M})$ and $\alpha$-L-guluronate $(\mathrm{G})$ with the following three types of block arrangement: 
polymannuronate (polyM), polyguluronate (polyG), and alternating random regions. ${ }^{1}$ The polysaccharide is abundant in brown seaweeds as a major cell wall matrix, while certain bacteria are also reported to be employed in biological alginate production. ${ }^{2}$ With its viscosity and gel-forming properties, alginate has drawn the attention of researchers on the aspect of their applications in food and pharmaceutical areas as food additives and drug carriers, respectively., ${ }^{3,4}$ Recent studies have also revealed the biological and physiological functions of brown seaweed-derived alginate oligosaccharides in stimulating human endothelial cells and in inducing plant growth. ${ }^{5}$ Distinct from polysaccharides (e.g., starch and cellulose) obtained from terrestrial plants, alginate is regarded as a potential marine biomass for biofuel production, since alginate is readily extracted from brown seaweeds at a mild alkaline $\mathrm{pH}$ and causes no serious competing interests for food stuffs. ${ }^{6}$ For certain alginate-producing bacteria, for example Pseudomonas aeruginosa, alginate as a component of bacterial biofilm plays crucial roles during cystic fibrosis infection. ${ }^{7}$ The biofilm produced by these bacteria contributes to protecting them from detrimental environments and confers drug resistance on the bacterial cells. Therefore, degradation and/or modification of alginate are considered to be important for biotechnological applications and for the medical treatment of biofilm-dependent infection.

Among alginate-assimilating bacteria, a gram-negative Sphingomonas sp. strain A1 is found to be distinctive, in which the bacterial cells directly incorporate the polysaccharide into the cytoplasm through a cell-surface mouth-like pit and a cytoplasmic membrane ABC transporter. ${ }^{8,9}$ The pit functions as a funnel for accumulating external alginates on the cell surface. The incorporated alginate is subsequently degraded by alginate lyases in the cytoplasm. Previous proteomic studies 
on cell-surface proteins reveal their functions as follows: Algp5 and Algp6 are alginate receptors, Alp7 and Algp8 are alginate-binding proteins, and Algp1-Algp4 are putative ferric ion-chelated alginate transporters. ${ }^{10}$

Genetically engineered strain A1 cells produce bioethanol from alginate. ${ }^{11}$ Clarification of alginate recognition mechanism by strain A1 is expected to construct modified bacterial cells with enhanced ability to produce ethanol. A cell-surface protein, Algp7, has been shown to have the ability to bind alginate at neutral $\mathrm{pH}$, and is considered to function as an alginate concentrator in pits. ${ }^{12}$ Based on its primary structure, Algp7 has been assigned to the EfeO family, and is encoded downstream of the efeUOB operon in the genome of strain $\mathrm{A} 1 .^{13} \mathrm{~A}$ tripartite EfeUOB has been genetically identified to act as an iron importer at low $\mathrm{pH}$ in gram-negative bacteria. ${ }^{14}$ EfeO has an N-terminal cupredoxin-like domain and a C-terminal M_75 peptidase-like domain; however, Algp7 lacks the cupredoxin-like domain at the N-terminus. ${ }^{15}$ Therefore, Algp7 contains only the M_75 peptidase domain. The high affinity of alginate with iron suggests Algp7 may bind iron as well as alginate.

Of late, the crystal structure of Algp7 has been determined at $2.1 \AA$ resolution; ${ }^{15}$ however, little knowledge about the interaction of Algp7 with alginate has been accumulated. This paper reports the results of X-ray crystallography, computational modeling with site-directed mutagenesis, and spectrometry-based alginate-binding assays aimed at elucidating the relationship between the structure and function of the cell surface alginate-binding protein Algp7.

\section{Results}

\subsection{Crystal structure of Algp7}


To gain structural insight into alginate binding by bacterial cell-surface protein, crystallization of Algp7 in the presence of various alginate-derived oligosaccharides, including G, GG, GGG, M, MM, and MMM, was performed. Previous evidence suggested that Algp7 can bind and release alginate polysaccharide, ${ }^{12}$ although alginate polysaccharide, as a macromolecule, is thought to be unsuitable for co-crystallization. In this experiment, alginate oligosaccharides prepared using acid hydrolysis were used alternatively to provide Algp7 crystal in a complex with a ligand.

Algp7 was crystallized in a drop solution containing $10 \mathrm{mM}$ of alginate oligosaccharide, and the crystal was formed for one week in a droplet containing mannuronate trisaccharide (MMM). Diffraction images were collected at up to $1.99 \AA$ resolution, providing higher resolution than previously used for Algp7; nonetheless, no oligosaccharides were included in the crystal structure (Table 1). The overall structure of Algp7 in this report corresponds well to the former determined structure (root mean square deviation, r.m.s.d. $=0.26$ ), showing that the protein has two up-and-down four-helix bundles (Fig. 1A). Though no atomic coordinates for the oligosaccharide were found for the present crystal structure, the Algp7 crystal diffracted to a higher resolution (1.99 $\AA$ ) was obtained with a shorter time for crystallization (one week at $20^{\circ} \mathrm{C}$ ). This result may be considered as a thermostability effect of oligosaccharides on the Algp7 molecule, reflected in improving the resolution of the crystal structure and reducing the time required for crystallization.

\subsection{Computational modeling}

Although many attempts at co-crystallization of Algp7 with various alginate oligosaccharides have been made, a complex of Algp7 with those ligands could not be obtained. Owing to the absence of an experimentally derived crystal structure of Algp7 
in complex with alginate oligosaccharides, computer-based studies using molecular docking have been performed to calculate the possible binding sites of the protein. Molecular docking has potential ability to identify binding sites because this method can build a reliable complex structure of target protein/ligands with reproducibility. ${ }^{16}$ Accordingly, AutoDock program, a widely used automated docking tool, ${ }^{17}$ was then applied in these studies to predict the location of the Algp7 binding site for alginate.

To establish whether AutoDock has the ability to reproduce protein/ligand complex structure, a preliminary test was performed in which a complex structure of AlgQ1 with mannuronate trisaccharide was used. AlgQ1 is a strain A1 periplasmic alginate-binding protein mediating the transport of alginate from the cell surface to the inner membrane, and the crystal structure of AlgQ1 in complex with alginate oligosaccharide has been reported. ${ }^{18}$ Atomic coordinates of trisaccharide (MMM) were excluded from AlgQ1/MMM [Protein Data Bank (PDB) ID: 3VLU] to use as ligand and atomic coordinates for apo-AlgQ1 was taken from PDB ID: 1Y3Q. Compared with the crystal structure of AlgQ1/MMM, the result from docking simulation of AlgQ1 with alginate oligosaccharide (MMM) showed that the ligand was closely accommodated at the binding site determined by X-ray crystallography, though the conformation of the ligand was partially different (data not shown). This docking simulation test demonstrated that AutoDock program could reliably find the binding site for alginate oligosaccharides on a target protein without prior knowledge of the binding site.

To probe the binding site of alginate on protein Algp7, the following two ligands were selected: a trisaccharide (MMM) derived from AlgQ1/MMM and a tetrasaccharide ( $\Delta \mathrm{M} 1-\mathrm{M} 2-\mathrm{G} 3-\mathrm{M} 4)$ from AlgQ1/DM1-M2-G3-M4. The atomic coordinates of the tetrasaccharide were excluded from AlgQ1/AM1-M2-G3-M4 (PDB ID: 1Y3P). A 
tetrasaccharide with unsaturated mannuronate $(\Delta \mathrm{M})$ at the nonreducing terminus is obtained from alginate via an alginate lyase reaction but not via hydrolysis. ${ }^{19}$ Each ligand was imported to Algp7 and the interaction between protein and ligand was calculated for the whole protein target using default parameters. In silico docking simulation resulted in three possible binding sites for alginate oligosaccharide, designated as models 1, 2, and 3 (Fig. 1C-E). Based on the constructed Algp7/oligosaccharide (tri and tetrasaccharide) models, the interaction of Algp7 residues with oligosaccharides via hydrogen bond formation is presented in Table 2. Considering the list, charged residues Lys68, Lys69, Glu194, and Asn221 were found to be potential candidates for alginate binding. To test the validity of these predicted residues, site-directed mutagenesis of these four residues was then performed and the effect of each mutation on alginate binding was functionally characterized.

\subsection{Characterization of Algp7 for alginate binding}

To investigate the predicted residues involved in alginate binding, mutant Algp7 constructs were generated using site-directed mutagenesis. Lys68, Lys69, Glu194, and Asn221 were substituted with Ala to characterize the effect of charged side chains on alginate binding. In addition, a double mutant K68A/K69A was constructed to verify the effect of positively charged cluster on the molecular surface of Algp7 observed on the constructed model (Fig. 1C). Mutant Algp7 constructs were expressed in E. coli cells with the same expression system as the wild type (pET44a-Algp7) and purified using the same procedures. The validation of the protein structure folding is described in the following section.

The alginate binding ability of the wild type and of each mutant construct of Algp7 was investigated using UV absorption spectroscopy, a spectroscopic technique for 
observing UV absorption change accompanying ligand binding. This method is rapid, simple, and requires only a spectrophotometer for detecting UV absorption. To determine whether UV absorption spectroscopy is appropriate to measure protein/ligand interaction, the wild-type Algp7 was tested with several saccharides. A UV absorption change was observed when alginate was added to protein solution, whereas no significant change was detected when other acidic polysaccharides such as pectin and heparin were used (Fig. 2A). The results supported previously published data indicating that Algp7 specifically binds alginate. Determination of the dissociation constant $K_{\mathrm{d}}$ of Algp7 for alginate was then performed using the same experiment. UV absorption changes, presumably reflecting differences in the environment of the protein resulting from ligand binding, were used to follow the binding of alginate. Because spectral differences depending on alginate concentration gave a saturation curve, the dissociation constant $K_{d}$ was determined by nonlinear curve fitting. The spectroscopic measurement revealed that Algp7 bound alginate with an estimated $K_{\mathrm{d}}$ of $7.19 \times 10^{-7} \mathrm{M}$ at $30^{\circ} \mathrm{C}$ (Fig. 2B).

Using this method, interaction between Algp7 mutants and alginate was examined to identify mutant constructs harboring a mutation at candidate amino acid residues identified by docking simulation. Replacement of a neutral side chain (Ala) at the positions of Lys68, Glu194, and Ans221 slightly affected UV absorption; however, $K_{\mathrm{d}}$ for alginate of the variants was comparable to that of the wild-type Algp7 (Table 3). In contrast, replacement of Lys with Ala at position 69, and substitution of Lys68 and Lys69 with Ala as a double mutant, contributed to a decrease in binding affinity (Fig. 2B). K69A led to an approximately 13-fold decrease in $K_{\mathrm{d}}$, whereas K68A/K69A did not give a saturation curve. Because of the decline in the binding ability for alginate of 
the double mutant K68A/K69A, $K_{d}$ for this mutant construct for alginate could not be determined (but at least a 20-fold decrease was considered.). The above results indicated the involvement of Lys68/Lys69 in binding alginate.

\subsection{Application of differential scanning fluorimetry for ligand binding analysis}

Differential scanning fluorimetry (DSF) was performed to further investigate the interaction of Algp7 and its mutants with alginate derivatives. DSF is a method used to identify small-molecule ligands that bind and stabilize proteins.$^{20}$ The method uses a sensitive fluorescence dye (SYPRO Orange) to monitor the thermostability change of a protein associating with a ligand, and the midpoint of the fluorescence transition profile is defined as the melting temperature $\left(T_{\mathrm{m}}\right)$. Several papers describing the characterization using DSF analysis of interactions between proteins and saccharides have been published. ${ }^{21,22}$

Alginate-derived oligosaccharides prepared from G-rich alginate saccharides, G, GG, GGG, and GGGG, were used as substrate ligands. Algp7 was primarily examined for the ability to bind alginate derivatives at a range of concentrations of 0 to $100 \mathrm{mM}$, and the $T_{\mathrm{m}}$ of Algp7 in the absence and presence of ligands (alginate oligosaccharides) was determined as the inflection midpoint of the increase in the fluorescence profile. The change $\left(\Delta T_{\mathrm{m}}\right)$ in melting temperature obtained in the presence of ligands relative to that obtained in the absence of ligands were plotted against ligand concentrations (Fig. 3A). Only the $T_{\mathrm{m}}$ of GGG at the concentration of $100 \mathrm{mM}$ could not be observed. The results demonstrated an increase in thermostability of Algp7 as the concentration of alginate-oligosaccharides was increased, suggesting a concentration-dependent effect of these oligosaccharides on the thermostability of Algp7.

Because DSF was suitable for evaluating the interaction between Algp7 and alginate 
oligosaccharides, the ability of the double mutant K68A/K69A for alginate oligosaccharide binding was then examined. The K68A/K69A mutant was tested against G, GG, GGG, and GGGG at a concentration of $20 \mathrm{mM}$. In the presence of $\mathrm{G}$ and GG as ligand, no significant positive thermal shifts were observed in the fluorescence profiles of K68A/K69A mutant. In contrast, the fluorescence profile of the K68A/K69A mutant in the presence of GGG and GGGG clearly shifted to a higher temperature, but showed a significant decrease in $\Delta T_{\mathrm{m}}$ compared with wild-type Algp7 (Fig. 3B-D). Recently, the DSF method has been demonstrated to be applicable for not only ligand binding assay but also investigation of the protein structure folding. ${ }^{20,23}$ It is known that if a protein is not folded and/or is partially unfolded, no transition will be observed or high initial fluorescence intensity will be exhibited in the beginning stage of the denaturation curve. ${ }^{20}$ However, there was no significant difference observed in the form of the fluorescence profile between the wild-type Algp7 and the double mutant K68A/K69A (Fig. 3C), indicating that both proteins were properly folded and the alginate-binding ability of the mutant was influenced by mutation, not misfolding.

The DSF fluorescence profile and deduced $\Delta T_{\mathrm{m}}$ indicated that the ability of the K68A/K69A mutant to bind alginate-derived oligosaccharides significantly decreased compared with wild-type Algp7. These results suggested that Lys68/Lys69 residues may be significant for binding to alginate oligosaccharides. Although alginate was not used as a ligand in this experiment because of its interference in binding of dye to protein, the observed results were consistent with the UV absorption spectra.

\section{Discussion}

It is commonly believed that interaction between a charged polysaccharide and a 
protein molecule is primarily driven by electrostatic forces. ${ }^{24}$ The negatively charged acidic polysaccharides thus tend to have an ability to interact with positively charged region of protein molecule. Based on the constructed Algp7/alginate oligosaccharide complex model, charged residues Lys68 and Lys69 were found to be interesting because they are neighboring residues and can form a positively charged cluster on the Algp7 molecule. The loss of positively charged surface on the K68A/K69A mutant led to a decrease in the ability to bind alginate, as seen in the results from UV absorption difference spectroscopy and DSF. Protein/carbohydrate interactions have common features including the optimization of hydrogen bonds and stacking interactions between aromatic residues and pyranose rings of sugars. ${ }^{25,26}$ Lys68 and Lys69 were found to form hydrogen bonds with alginate oligosaccharide in simulations. Furthermore, the nearby Trp187 residue was also observed to make contact with the face of the sugar ring (Fig. 1C, F). In many reported protein/carbohydrate complexes, tryptophan residues are crucial for stacking interaction. This stacking interaction helps stabilize the complexes. ${ }^{26}$ Protein is commonly measured at an optical absorbance of $280 \mathrm{~nm}$. Three aromatic amino acid side chains, tryptophan, tyrosine, and phenylalanine are the important chromophores in the near ultraviolet, having a significant absorption band between 250 and $320 \mathrm{~nm} .{ }^{27}$ The spectral change derived from tryptophan has been used as a probe to monitor protein conformational change. ${ }^{28}$ Algp7 has only one tryptophan residue at the 187 position; therefore, a UV absorption change derived from Trp187 associating with alginate binding may reflect the perturbation of Trp187 and may be used to follow alginate binding via Algp7. Structural details in interaction between Algp7 and alginate oligosaccharide seen in the constructed model were well explained by the experimental results, indicating the reliability of the predicted binding 
site from docking simulation.

\section{Conclusion}

The interaction of strain A1 cell-surface EfeO-like protein Algp7 with oligoalginate was simulated. UV absorption difference spectroscopy and DSF using Algp7 mutants indicated that Lys68 and Lys69 residues probably play an important role in binding alginate. The negatively charged alginate seems to interact with this positively charged cluster.

\section{Experimental}

\subsection{Materials}

Sodium alginate and heparin sodium salt were purchased from Nacalai Tesque. Pectin was purchased from Sigma-Aldrich. Synthetic oligonucleotides used for site-directed mutagenesis were purchased from Hokkaido System Science. KOD Plus Neo, dNTPs, and $\mathrm{MgSO}_{4}$ for PCR were purchased from Toyobo.

\subsection{Protein expression and purification}

Escherichia coli strain BL21(DE3) (Novagen) was used as a host for the expression of Algp7. E. coli cells were aerobically precultured at $30^{\circ} \mathrm{C}$ in Luria-Bertani (LB) medium ${ }^{29}$ containing $0.1 \mathrm{mg} / \mathrm{mL}$ sodium ampicillin to an optical density at $600 \mathrm{~nm}$ $\left(\mathrm{OD}_{600}\right)$ of 0.5 . Expression of the target gene was induced by addition of isopropyl $\beta$-D-thiogalactopyranoside $(0.1 \mathrm{mM})$, and the cells were further cultured at $16^{\circ} \mathrm{C}$ for 44 h. Unless otherwise specified, the following operations were performed at $0^{\circ} \mathrm{C}-4^{\circ} \mathrm{C}$. $\mathrm{E}$. coli cells harboring pET44a-Algp $7^{12}$ were grown in $13.5 \mathrm{~L}$ of LB medium (1.5 L/flask), harvested by centrifugation at $6,000 \mathrm{~g}$ and $4^{\circ} \mathrm{C}$ for $5 \mathrm{~min}$, washed with $20 \mathrm{mM}$ Tris- $\mathrm{HCl}$ 
(pH7.5), and resuspended in the same buffer. The cells were ultrasonically disrupted (Insonator Model 201M, Kubota) at $0^{\circ} \mathrm{C}$ and $9 \mathrm{kHz}$ for $20 \mathrm{~min}$, and the soluble fraction obtained after centrifugation at $20,000 \mathrm{~g}$ and $4^{\circ} \mathrm{C}$ for 20 min was used as the cell extract. Algp7 was purified from the cell extract by affinity chromatography [TALON column $(1.0 \times 10 \mathrm{~cm})$; Clontech] and ion-exchange chromatography [Toyopearl SuperQ-650M column $(1.0 \times 10 \mathrm{~cm})$; Tosoh]. The eluted protein Algp7 was dialyzed against $20 \mathrm{mM}$ Tris-HCl (pH 7.5) overnight, and the dialysate was used as purified Algp7. The protein sample was analyzed using SDS-PAGE ${ }^{30}$ to confirm homogeneity.

\subsection{Crystallization and $X$-ray diffraction}

Algp7 (8 mg/mL) was co-crystallized in the presence of various alginate oligosaccharides at $20^{\circ} \mathrm{C}$ using the hanging-drop vapor diffusion method. The mixture [18\% polyethylene glycol monomethyl ether 5,000, $0.1 \mathrm{M}$ Tris- $\mathrm{HCl}$ (pH 7.37), and 0.2 M lithium sulfate] (500 $\mu \mathrm{L})$ was used as a reservoir solution. A droplet was prepared by mixing $3 \mu \mathrm{L}$ of protein solution containing alginate oligosaccharide with an equal volume of the reservoir solution. The crystal was picked up with a mounted nylon loop (Hampton Research), and placed directly into a cold nitrogen-gas stream at $-173^{\circ} \mathrm{C}$. X-ray diffraction images were collected with a Quantum 315 charge-coupled device detector (ADSC) and synchrotron radiation at a wavelength of $1.00 \AA$ at the BL-38B1 station of SPring-8 (Japan). The diffraction data were processed using HKL2000. ${ }^{31}$

\subsection{Structure determination and refinement}

The crystal structure of Algp7 was determined through molecular replacement with

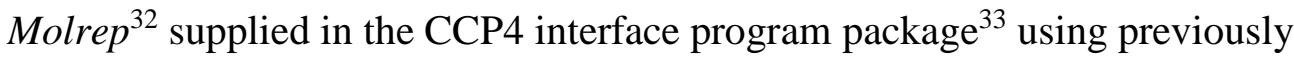
determined coordinates of Algp7 (PDB ID: 3AT7) as an initial model and refined with PHENIX.${ }^{34}$ Randomly selected reflections (5\%) were excluded from refinement and 
used to calculate $R_{\text {free. }}$ After each refinement cycle, the model was manually adjusted using WinCoot. ${ }^{35}$ Water molecules were incorporated where the difference in density exceeded $3.0 \sigma$ above the mean, and the $2 F_{0}-F_{c}$ map showed a density of more than $1.2 \sigma$. Figures for protein structures were prepared using $P y M O L .{ }^{36}$ Coordinates used in this work were taken from PDB of the Research Collaboratory for Structural Bioinformatics. ${ }^{37}$

\subsection{Preparation of alginate derivatives}

M- and G-rich saccharides were separated from seaweed alginate as described previously. ${ }^{38} \mathrm{M}$ - and G-rich alginate oligosaccharides were prepared by acid hydrolysis and gel filtration. The content of G in G-rich saccharides seems to be over $90 \%{ }^{21}$ In brief, G-rich saccharides ( $0.3 \%$ solution) were hydrolyzed with $7 \mathrm{mM} \mathrm{HCl}$ at $100^{\circ} \mathrm{C}$ for approximately $20 \mathrm{~h}$. After neutralization with $\mathrm{NaOH}$, the solutions were lyophilized and loaded onto gel filtration columns [Biogel P-2 column $(1.5 \times 140 \mathrm{~cm})$; Bio-Rad]. Hydrolyzed oligosaccharides were separated and purified to each saccharide; monosaccharide (G), disaccharide (GG), trisaccharide (GGG), and tetrasaccharide (GGGG). The alginate oligosaccharides were confirmed as homogeneous using thin-layer chromatography (TLC) developed with a solvent system of $n$-butanol/formic acid/water, 4:6:1 (v/v). ${ }^{39}$ The TLC plates were visualized by heating at $110^{\circ} \mathrm{C}$ for $5 \mathrm{~min}$ after spraying with $10 \%(\mathrm{v} / \mathrm{v})$ sulfuric acid in ethanol. M-rich oligosaccharides (monosaccharide, $\mathrm{M}$; disaccharide MM; trisaccharide, MMM) were prepared by the same process.

\subsection{Molecular modeling}

Three-dimensional structures of proteins were obtained from PDB (PDB ID: 3AT7 for Algp7, PDB ID: 1Y3Q for apo-AlgQ1, PDB ID: 3VLU for AlgQ1/MMM, and PDB 
ID: 1Y3P for AlgQ1/DM1-M2-G3-M4). Coordinates of ligands were extracted from the above mentioned complex structures. The possible binding sites of ligands to Algp7 were predicted using the AutoDock package. ${ }^{17}$ A model of the protein/ligand complexes was built by importing the structures of alginate oligosaccharides into the protein structures after removing all water molecules from the crystal structures. Polar hydrogen atoms were added and grid maps were generated with AutoGrid ${ }^{17}$ for the whole protein target. Interactions between proteins and ligands were calculated for the possible conformation of the ligands binding to proteins by means of a Lamarckian genetic algorithm using default parameters. All docking processes were run under the Linux operating system.

\subsection{UV absorption spectroscopy}

Interactions between proteins and polysaccharides (alginate, heparin, and pectin) were evaluated using UV absorption spectroscopy. UV absorption spectra accompanying ligand binding were observed at $\mathrm{pH} 7.5$ [10 mM sodium 4-(2-hydroxyethyl)-1-piperazineethanesulfonic acid (HEPES)] and $30^{\circ} \mathrm{C}$ with a Shimadzu UV-2600 spectrophotometer.

\subsection{DSF}

Binding analysis between proteins and alginate-derived oligosaccharides was performed using $\mathrm{DSF}^{20}$ with a MyiQ2 real-time PCR instrument (Bio-Rad). The method is based on thermal shift assay monitored by fluorescent dye [SYPRO Orange; Invitrogen]. Algp7 and its mutants were added to a final concentration of $18 \mu \mathrm{M}$ in a 20 $\mu \mathrm{L}$ total volume of reaction mixture consisting of $20 \mathrm{mM}$ Tris- $\mathrm{HCl}$ ( $\mathrm{pH}$ 7.5), each oligosaccharide (0-100 mM), and freshly prepared SYPRO Orange (1000-fold diluted). The change of fluorescence emitted from SYPRO Orange upon binding to the denatured 
protein was monitored using a filter provided in the PCR instrument (excitation at 492 $\mathrm{nm}$ and emission at $610 \mathrm{~nm}$ ). The temperature of the heating cycle was increased from $25^{\circ} \mathrm{C}$ to $95^{\circ} \mathrm{C}$ in $0.5^{\circ} \mathrm{C}$ increments (10s/cycle) for a total of 141 cycles. The obtained fluorescence data were collected and the midpoint of the first derivative of the relative fluorescence unit (RFU) curve was calculated to provide the $T_{\mathrm{m}}$ using the software installed on the instrument.

\subsection{Site-directed mutagenesis}

Mutations of Algp7 were constructed by PCR on the pET44a-Algp7 plasmid (template) with overlapping primers containing the mutation. After temperature cycling, the original plasmid was digested with DpnI, and the PCR mixture was then transformed into E. coli cells. After isolation of the plasmids, the mutations were verified using DNA sequence analysis and then transformed into E. coli cells BL21(DE3) for protein expression in the same way as the wild type.

The primers used in the experiment were as follows:

K68A sense:

5'-GCGGGCGACGTCGCCAAGGCGGCTAAGCTCTTCGCCTCTACGCGC-3'; K68A antisense:

5'-GCGCGTAGAGGCGAAGAGCTT $\underline{A G C C G C C T T G G C G A C G T C G C C C G C-3 ' ; ~}$ K69A sense:

5’-GCGGGCGACGTCGCCAAGGCGAAGGCACTCTTCGCCTCTACGCGC-3’; K69A antisense:

5'-GCGCGTAGAGGCGAAGAGTGCCTTCGCCTTGGCGACGTCGCCCGC-3'; K68A/K69A sense:

5’-GCGGGCGACGTCGCCAAGGCGGCTGCACTCTTCGCCTCTACGCGC-3’; 
K68A/K69A antisense:

5'-GCGCGTAGAGGCGAAGAGTGCAGCCGCCTTGGCGACGTCGCCCGC -3'; E194A sense:

5'-CTGTGGGACTTCCAGGCCAATTTCGCGGGCGCCAAGAAGATCGTC-3'; E194A antisense:

5'-GACGATCTTCTTGGCGCCCGCGAAATTGGCCTGGAAGTCCCACAG -3'; N221A sense:

5'-GCCGACAAGGTAGATGCCGCGTTCGATACGGTCTTCAAGACACTG- 3'; N221A antisense:

5'-CAGTGTCTTGAAGACCGTATCGAACGCGGCATCTACCTTGTCGGC -3'. The site of the mutation is indicated by underlining.

\section{Accession codes}

Complete crystallographic data for the structural analysis have been deposited with the Protein Data Bank, PDB: 3WSC.

\section{Acknowledgements}

This work was supported in part by Grants-in-Aid for Scientific Research (W.H.) (No. 2666062) from the Japan Society for the Promotion of Science, by the Targeted Proteins Research Program (W.H.) (No. 07050217) from the Ministry of Education, Culture, Sports, Science and Technology of Japan and by the program (K.M.) (No. 08063738) for the Promotion of Basic Research Activities for Innovative Bioscience (PROBRAIN) in Japan. We thank Drs. S. Baba and N. Mizuno from the Japan Synchrotron Radiation Research Institute (JASRI) for their kind help in data collection. Diffraction data for 
crystals were collected at the BL-38B1 (Hyogo, Japan), with the approval (2012B1265 and 2013A1106) of JASRI.

\section{References}

1. Gacesa, P. Carbohydr. Polym. 1988, 8, 161-1822.

2. Sabra, W.; Zeng, A.P.; Deckwer, W.D. Applied Microbiol. Biotechnol. 2001, 56, 315-325.

3. $\quad$ Aliste, A.J.; Vieira, F.F.; Del Mastro, N.L. Radiat. Phys. Chem. 2000, 57, 305-308.

4. Skaugrud, Ø.; Hagen, A.; Borgersen, B.; Dornish, M. Biotechnol. Genet. Eng. 1999, 16, 23-40.

5. $\quad$ Kawada, A.; Hiura, N.; Tajima, S.; Takahara, H. Arch. Dermatol. Res. 1999, 291, $542-547$.

6. $\quad$ Stokstad, E. Science 2012, 335, 273.

7. $\quad$ May, T.B.; Chakrabarty, A.M. Trends Microbiol. 1994, 2, 151-157.

8. $\quad$ Mishima, Y.; Momma, K.; Hashimoto, W.; Mikami, B.; Murata, K. FEMS Microbiol. Lett. 2001, 204, 215-221.

9. Murata, K.; Kawai, S.; Mikami, B.; Hashimoto, W. Biosci. Biotech. Bioch. 2008, 72, 265-277.

10. Hashimoto, W.; He, J.; Wada, Y.; Nankai, H.; Mikami, B.; Murata, K. Biochemistry 2005, 44, 13783-13794.

11. Takeda, H.; Yoneyama, F.; Kawai, S.; Hashimoto, W.; Murata, K. Energy Environ. Sci. 2011, 4, 2575-2581.

12. He, J.; Ochiai, A.; Fukuda, Y.; Hashimoto, W.; Murata, K. FEMS Microbiol. 
Lett. 2008, 288, 211-226.

13. Cao, J.; Woodhall, M.R.; Alvarez, J.; Cartron, M.L.; Andrews, S.C. Mol. Microbiol. 2007, 65, 857-875.

14. Rajasekaran, M.B.; Nilapwar, S.; Andrews, S.C.; Watson, K.A. Biometals 2010, 23, 1-17.

15. Maruyama, Y.; Ochiai, A.; Mikami, B.; Hashimoto, W.; Murata, K. Biochem. Biophys. Res. Commun. 2011, 405, 411-416.

16. Hetényi, C.; Van der spoel, D. Protein Sci. 2002, 11, 1729-1737.

17. Morris, G.M.; Goodsell, D.S.; Halliday, R.S.; Huey, R.; Hart, W.E.; Belew, R.K.; Olson, A. J. J. Comput. Chem. 1998, 19, 1639-1662.

18. Momma, K.; Mishima, Y.; Hashimoto, W.; Mikami, B.; Murata, K. Biochemistry 2005, 44, 5053-5064.

19. Hashimoto, W.; Okamoto, M.; Hisano, T.; Momma, K.; Murata, K. J. Ferment. Bioeng. 1998, 86, 236-238.

20. Niesen, F.H.; Berglund, H.; Vedadi, M. Nat. Protoc. 2007, 2, 2212-2221.

21. Nishitani, Y.; Maruyama, Y.; Itoh, T.; Mikami, B.; Hashimoto, W.; Murata, K. Biochemistry 2012, 51, 3622-3633.

22. Uniewicz, K.A.; Ori, A.; Xu, R.; Ahmed, Y.; Wilkinson, M.C.; Fernig, D.G.; Yates, E.A. Anal. Chem. 2010, 82, 3796-3802.

23. Vollrath, F.; Hawkins, N.; Porter, D.; Holland, C.; Boulet-Audet, M. Sci. Rep. 2014, 4, 5625.

24. Imeson, A.P.; Ledward, D.A.; Mitchell, J.R. J. Sci. Food Agric.1977, 28, 661-668.

25. Rini, J.M. Annu. Rev. Biophys. Biomol. Struct. 1995, 24, 551-577. 
26. Vyas, N.K. Curr. Opin. Struct. Biol. 1991, 1, 732-740.

27. Goldfarb, A.R.; Saidel, L.J.; Mosovich, E. J. Biol. Chem. 1951, 193, 397-404.

28. Lin, S.W.; Sakmar, T.P. Biochemistry 1996, 35, 11149-11159.

29. Sambrook, J.; Fritsch, E.F.; Maniatis, T. Molecular cloning, A laboratory manual, $2^{\text {nd }}$ ed.; Cold Spring Harbor Laboratory Press: New York, 1989.

30. Laemmli, U.K. Nature 1970, 227, 680-685.

31. Otwinoski, Z.; Minor, W. Methods Enzymol. 1997, 276, 307-326.

32. Vagin, A.; Teplyakov, A. J. Appl. Crystallogr. 1997, 30, 1022-1025.

33. Collaborative Computational Project. Acta Crystallogr. 1994, D50, 760-763.

34. Adams, P.D.; Grosse-Kunstleve, R.W.; Hung, L-W.; Ioerger, T.R.; McCoy, A.J.; Moriarty, N.W.; Read, R.J.; Sacchettini, J.C.; Sauter, N.K.; Terwilliger, T.C. Acta Crystallogr. 2002, D58, 1948-1954.

35. Emsley, P.; Cowtan, K. Acta Crystallogr. 2004, D60, 2126-2132.

36. DeLano, W.L. The PyMol molecular graphics system, DeLano Scientific LLC: California, 2004.

37. Berman, H.M.; Westbrook, J.; Feng, Z.; Gilliland, G.; Bhat, T.N.; Weissig, H.; Shindyalov, I.N.; Bourne, P.E. Nucleic Acids Res. 2000, 28, 235-242.

38. Haug, A.; Larsen, B.; Smidsrod, O. Acta Chem. Scand. 1966, 20, 183-190.

39. Shimokawa, T.; Yoshida, S.; Kusakabe, I.; Takeuchi, T.; Murata, K.; Kobayashi, H. Carbohydr. Res. 1997, 304, 125-132. 


\section{Figure Legends}

Figure 1. Structure and candidate residues for alginate binding. (A) Overall structure of Algp7 determined using X-ray crystallography. Algp7 molecule is shown as a ribbon model (blue). (B) Overall electrostatic surface of Algp7. (C) Docking model with ligand-binding Lys68 and Lys69 (model 1). (D) Docking model with ligand-binding Glu194 (model 2). (E) Docking model with ligand-binding Asn221 (model 3). Alginate oligosaccharide molecules used as ligands are shown as stick models (carbon, light blue; oxygen, red). Side chains of candidate residues forming hydrogen bonds are colored green. (F) Electrostatic surface of docking model showing positively charged cluster (Lys68 and Lys69) and alginate oligosaccahride.

Figure 2. Algp7 binding to polysaccharides. Interaction between proteins and polysaccharides was evaluated using UV absorption spectroscopy. (A) Binding specificity of Algp7. Algp7 was tested with pectin, heparin, and alginate (each 50 $\mu \mathrm{g} / \mathrm{ml})$ at $\mathrm{pH} 7.5$ (HEPES-NaOH). UV absorption spectra showed substrate-binding specificity of Algp7 to alginate. (B) Saturation curve of alginate for Algp7 and its mutants. Algp7 mutants were tested with alginate at pH 7.5 (HEPES-NaOH). (rhombus; Algp7, plus; K68A, circle; K69A, square; E194A, triangle; N221A, multiple mark; K68A/K69A)

Figure 3. Binding analysis using differential scanning fluorimetry (DSF). (A) Concentration dependent effect of alginate-derived oligosaccharides on thermal stabilization of Algp7 (rhombus; Algp7 with G, square; Algp7 with GG, triangle; Algp7 with GGG, multiple mark; Algp7 with GGGG). (B) Effect of alginate-derived 
oligosaccharides on $\Delta T_{\mathrm{m}}$ of Algp7 (black), K68A/K69A (shaded), and N221A (blank).

(C) Left, fluorescence profile of Algp7 and K68A/K69A mutant with 20-mM GGGG.

Right, negative derivative curve plot obtained from the fluorescence profile. 

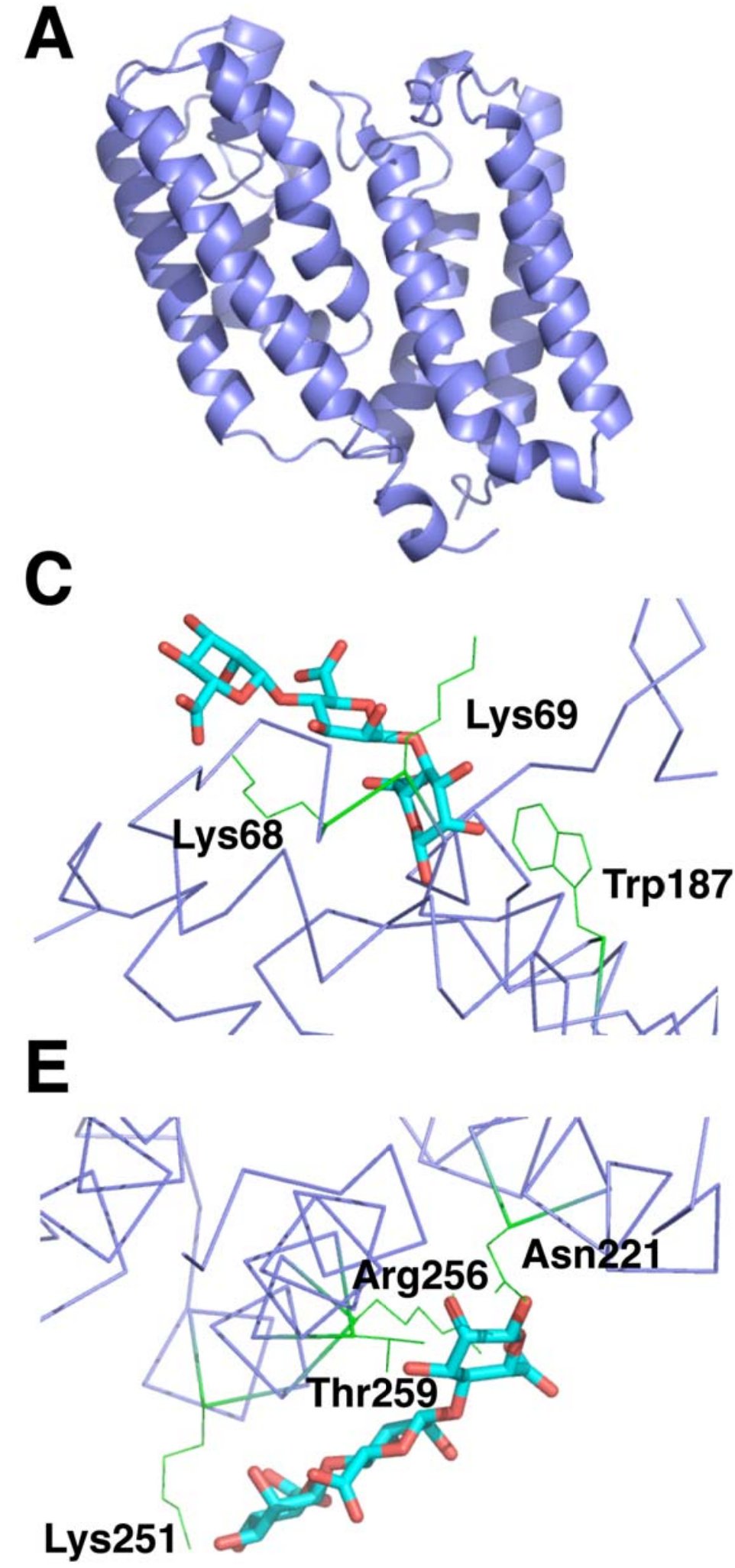

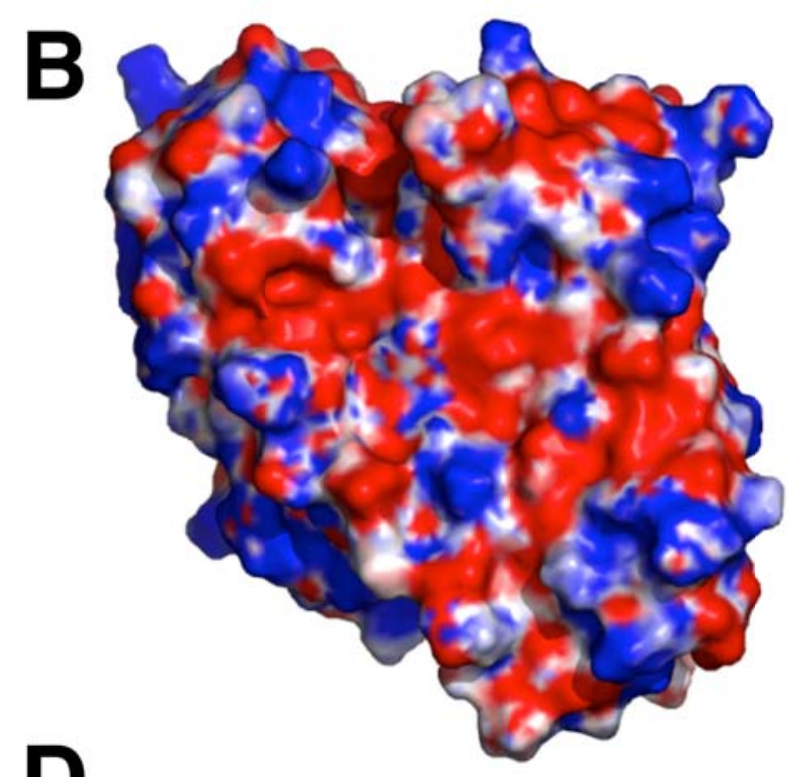

D

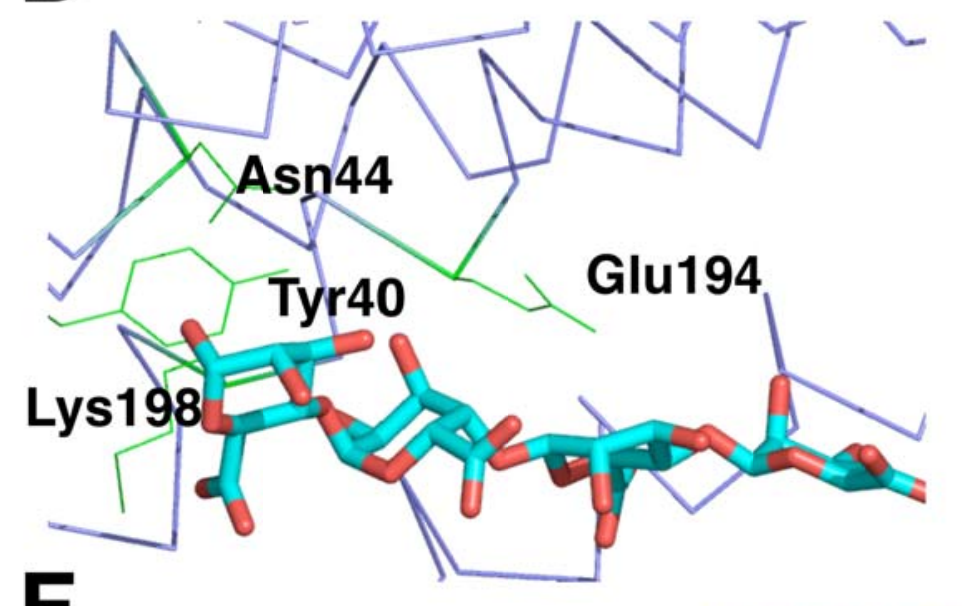

$\mathbf{F}$

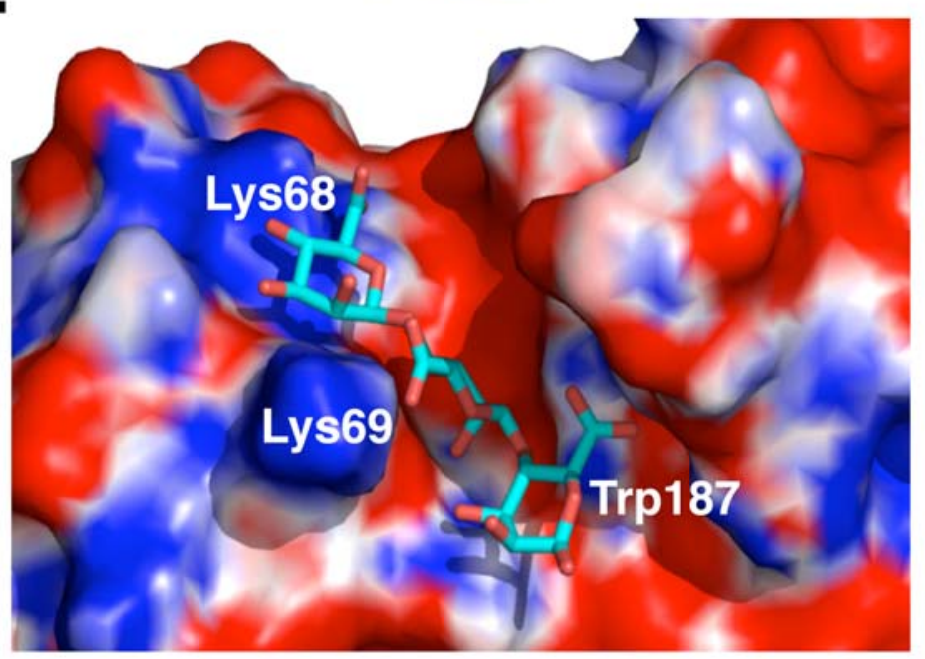


A

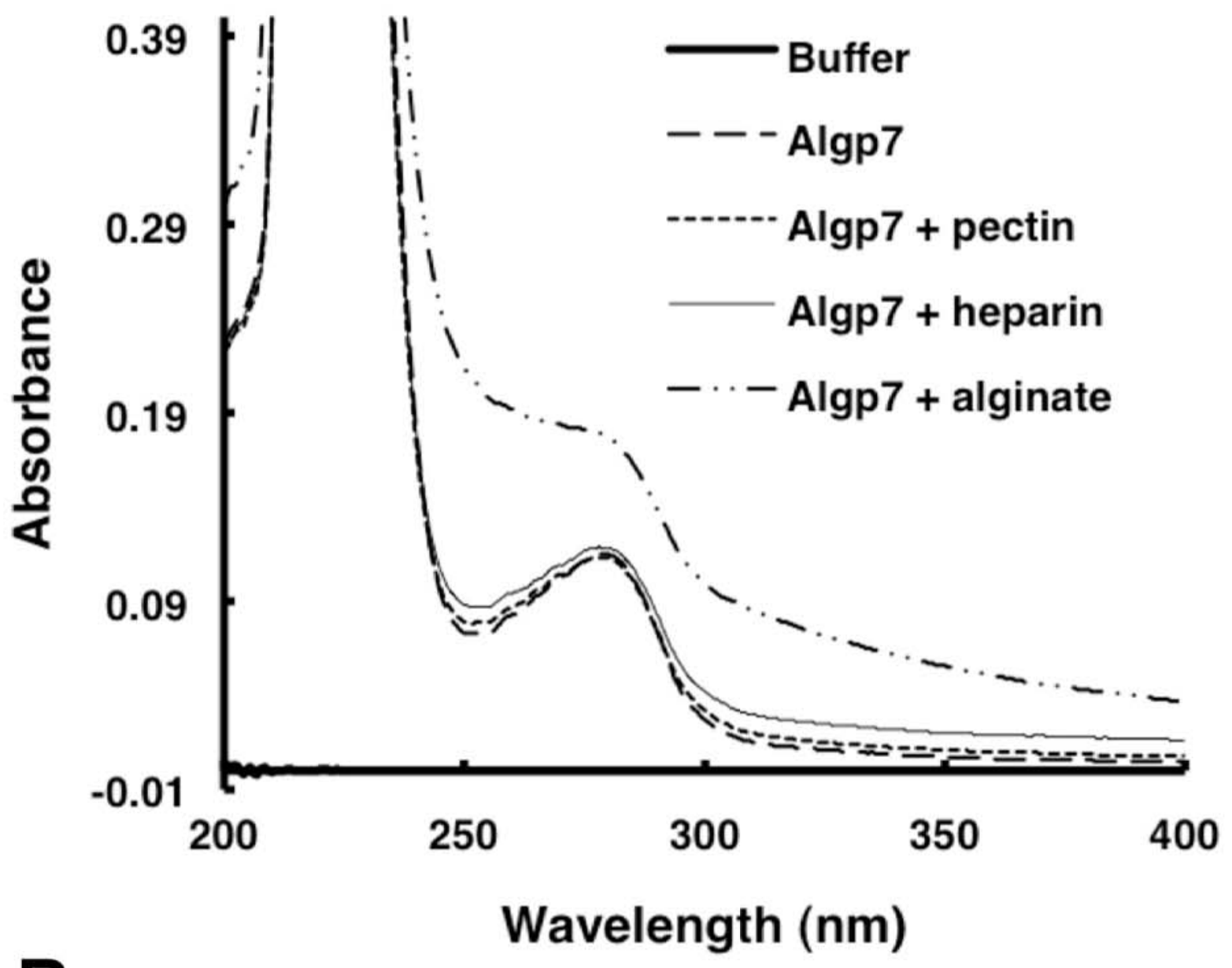

B

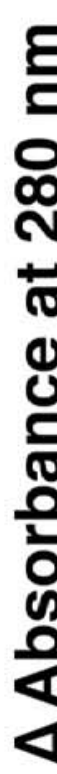
0.35
0.3
0.25
0.15
0.1
0.05

0.2

0
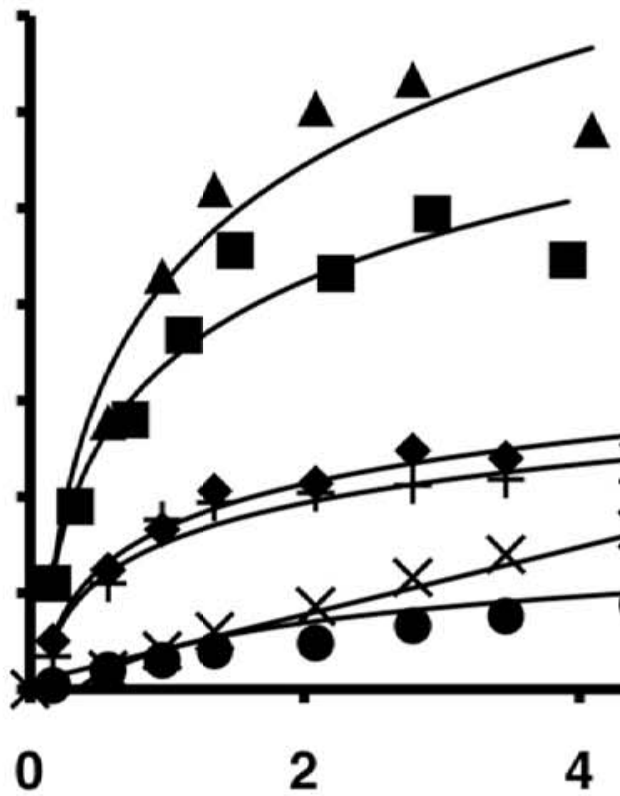

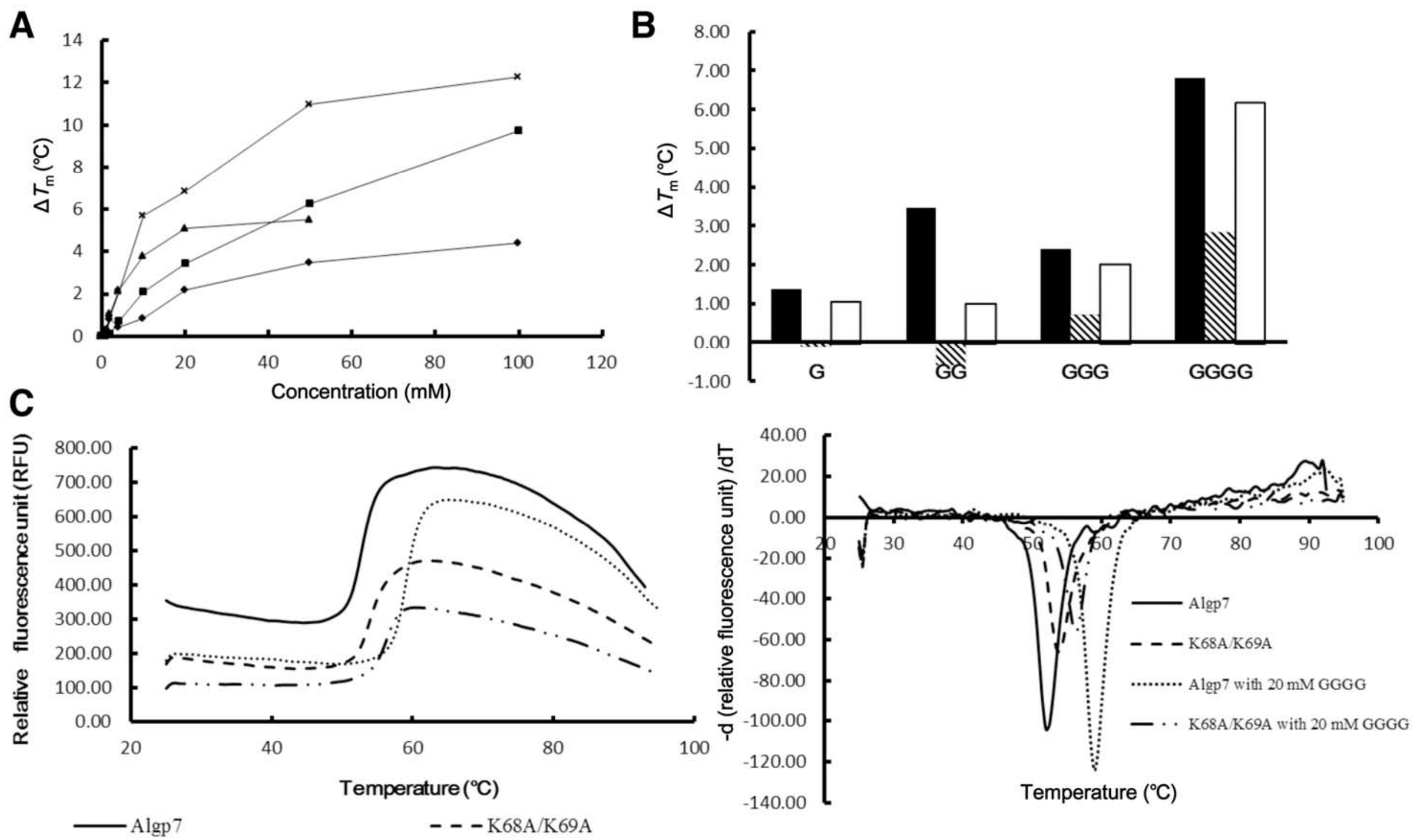

$---\mathrm{K} 68 \mathrm{~A} / \mathrm{K} 69 \mathrm{~A}$ 


\section{Table 1}

Data collection and refinement statistics

Algp7

Data collection

Wavelength $(\AA)$

1.00

Resolution range ( $\AA$ )

$50.00-1.99(2.07-1.99)^{\mathrm{a}}$

Space group

$P 2{ }_{1}{ }_{1} 2_{1}$

Unit-cell parameter $(\AA)$

$a=53.16, b=97.70, c=103.08$

Total observations

336508

Unique reflections

32152

Completeness (\%)

$98.00(100.00)$

$\mathrm{I} / \sigma(\mathrm{I})$

40.56 (8.50)

$R_{\text {merge }}$

$0.081(0.421)$

Refinement

$R_{\text {cryst }}$

0.20

$R_{\text {free }}$

0.24

No. of molecules per ASU

2

No. of non-hydrogen atoms

Proteins

Water molecules

Average B-factor $\left(\AA^{2}\right)$

r.m.s.d. from ideal

Bond length $(\AA)$

0.003

Bond angle (deg.)

0.75

Ramachandran plot (\%)

Favored

97.59

Allowed

2.41

Outliers

0

${ }^{a}$ Data for highest shells are given in parentheses.

${ }^{\mathrm{b}}$ ASU, asymmetric unit. 


\section{Table 2}

Hydrogen bond-forming residues and close contact residues with ligand from in silico Algp7/alginate-oligosaccharide model

\begin{tabular}{|c|c|c|}
\hline Model & H-bond residues & Close contact residues \\
\hline \multirow[t]{3}{*}{1} & Lys68 & Phe122 \\
\hline & Lys69 & Ala72 \\
\hline & Ser73 & Trp187 \\
\hline \multirow[t]{4}{*}{2} & Tyr40 & Gln190 \\
\hline & Asn44 & Phe226 \\
\hline & Glu194 & Arg233 \\
\hline & Lys198 & \\
\hline \multirow[t]{4}{*}{3} & Asn221 & Thr224 \\
\hline & Lys251 & Val252 \\
\hline & Arg256 & Gly255 \\
\hline & Thr259 & \\
\hline
\end{tabular}

Candidate amino acid residues for alginate binding are shown in bold. 


\section{Table 3}

Dissociation constants of Algp7 and its mutants with alginate

\begin{tabular}{cc}
\hline Proteins & Estimated $K_{\mathrm{d}}\left(\times 10^{-7} \mathrm{M}\right)$ \\
\hline Wild-type Algp7 & $7.19 \pm 4.58$ \\
K68A & $6.34 \pm 1.37$ \\
K69A & $95.03 \pm 38.89$ \\
K68A/K69A & N.D. \\
E194A & $5.79 \pm 1.48$ \\
N221A & $6.66 \pm 1.63$
\end{tabular}

Each value represents the average of triplicate individual experiments (mean \pm s.d.). 